Revue des patrimoines

$20 \mid 2013$

Les patrimoines de la traite négrière et de l'esclavage

\title{
Archives de plantations aux Antilles
}

Jacques Dion

\section{(2) OpenEdition}

Journals

Édition électronique

URL : http://journals.openedition.org/insitu/10103

DOI : 10.4000/insitu.10103

ISSN : 1630-7305

\section{Éditeur}

Ministère de la Culture

\section{Référence électronique}

Jacques Dion, «Archives de plantations aux Antilles », In Situ [En ligne], 20 | 2013, mis en ligne le 12 février 2013, consulté le 14 novembre 2019. URL : http://journals.openedition.org/insitu/10103 ; DOI 10.4000/insitu. 10103

Ce document a été généré automatiquement le 14 novembre 2019.

\section{(c) (i) (9)}

In Situ Revues des patrimoines est mis à disposition selon les termes de la licence Creative Commons Attribution - Pas d'Utilisation Commerciale - Pas de Modification 4.0 International. 


\title{
Archives de plantations aux Antilles
}

\author{
Jacques Dion
}

1 Les archives de plantations ou d'habitations constituent une source de première main : l'historien de l'esclavage Gabriel Debien en avait bien compris tout l'intérêt puisqu'une grande part de son œuvre, novatrice à bien des égards, est fondée sur ses découvertes de fonds privés et les enseignements qu'il put tirer de ces papiers. Leur précarité appelle à la vigilance.

2 Deux fonds d'archives récemment entrés dans les archives publiques nous permettent de mieux connaître la société esclavagiste des Antilles françaises au XIX ${ }^{\mathrm{e}}$ siècle.

3 La plantation coloniale, aussi dénommée habitation, produit sucre, café, indigo, coton...: elle constitue la base économique de la société esclavagiste des colonies françaises d'Amérique. L'historien Pierre Pluchon la définit comme une « unité agromanufacturière et capitaliste » formée de quatre éléments : le domaine agricole, la manufacture, le groupe humain et le cadre financier. Elle est inévitablement le fondement humain de la société créole ; c'est là que s'établissent les rapports entre les Noirs et les Blancs, rapports de force commandés par les exigences de la production, les gains à faire, la tranquillité à assurer. Ces rapports vont s'établir sur la longue durée.

4 La majorité des esclaves réside sur les plantations : la discipline y règne sous l'autorité du commandeur, aux ordres du gérant. La répartition des tâches et leur hiérarchie, le rythme des travaux, le commandement des hommes, la topographie même des habitations imposent un modèle de vie peu différencié d'une habitation à l'autre. Cette organisation sociale et spatiale se met en place dès la fin du XVII ${ }^{e}$ siècle et va perdurer jusqu'à l'abolition de 1848 .

5 Les habitations de taille moyenne (les plus nombreuses) comptent environ 200 esclaves, les plus vastes 1000 ou plus.

\section{Un propriétaire sur ses terres : le général Bertrand}

Le 22 décembre 1836, le Bélisaire quitte Cherbourg pour la Martinique emportant à son bord Henri Gatien, comte Bertrand ${ }^{1}$, âgé de 63 ans, accompagné de son fils Arthur, 
19 ans, natif de l'île de Sainte-Hélène. Le navire arrivera à Saint-Pierre de la Martinique le 22 janvier 1837.

7 En 1836, le général, ami fidèle de Napoléon $1^{\mathrm{er}}$, après une longue carrière, s'est retiré de la vie publique et gère, aidé de son frère, un vaste domaine près de Châteauroux dans l'Indre. Sa femme, Françoise Élisabeth de Dillon, meurt le 6 mars 1836, laissant en héritage deux habitations sucrières: les Salines (commune de Sainte-Anne) et les Coteaux (commune de Sainte-Luce). Fanny de Dillon était l'héritière de Marie Françoise Louise de Girardin et d'Arthur, comte de Dillon (1750-1794). Les Coteaux couvrent 284 ha, les Salines 116 ha ; on y compte près de 250 esclaves.

8 C'est afin de vendre ces biens que le général Bertrand arrive en Martinique: les propriétés sont vendues sur licitation et il s'en rend lui-même adjudicataire. Cette procédure lui permet de régler les dettes familiales. Le général rentre en France le 10 juillet 1839 sur le Majestueux qui arrive à Bordeaux le 16 août.

Cet épisode peu connu de la vie du général se révèle d'une particulière richesse: Bertrand, de retour dans l'Indre, constitue un épais dossier formé des lettres envoyées à son frère resté à Châteauroux, des lettres reçues pendant son séjour sur l'île, de nombreuses notes relatives à l'esclavage en forme de chapitres, d'une correspondance avec le baron Dupin.

10 L'intérêt que porte le général à ses affaires, sa perspicacité vont faire de lui un remarquable témoin. Le général réside aux Salines et c'est de là qu'il envoie à son frère de nombreuses lettres qui permettent de suivre son action avec une certaine régularité.

11 À son arrivée le 5 février 1837, il découvre un monde nouveau et étonnant. «En arrivant hier soir nous avons été entourés de tous les nègres et négresses de l'habitation et des négrillons tous fort contents de recevoir leur maître et le petit maître. Depuis longtemps ils n'avaient plus que des géreurs. Ils ont congé demain, comme la danse est leur plaisir ils ont demandé un violon. Depuis 4 à 6 ans ils préfèrent danser au violon des contredanses et ils abandonnent leurs danses au tambour. On leur donne du vin, de la farine, on tuera un petit bœuf. Le violon se paye une pièce d'or, $21 \mathrm{f} 60 \mathrm{c}$. Les nègres sont légèrement vêtus avec des toiles souvent en guenilles mais le dimanche ils ont chapeaux, gilets, pantalons, et les négresses sont aussi élégantes, des boucles d'oreilles d'or, madras, fichus, robes, bas et souliers. L'attelier (sic) des Salines passe pour un des meilleurs du quartier. Ils ont une industrie qui leur est particulière, une espèce de poterie dont ils fournissent toute l'île, ou du moins une grande partie ; on appelle canaries ces pots fabriqués avec de la terre des salines. »

12 Le général s'imprègne des méthodes de culture, du matériel et de la mécanisation, de l'organisation du travail. Les voies de communication, l'irrigation sollicitent son savoirfaire.

13 Le 17 février: «[...] Je vais tâcher de te mettre au courant du peu que j'ai vu de la culture de ce pays dont plusieurs choses me sont encore inconnues comme tu le concevras très bien. Depuis quelques années la charrue est adoptée dans ce pays, il y en a deux aux Salines; on en trouve également dans toutes les habitations voisines [...] À présent tout le terrain est labouré à la charrue ensuite on forme des sillons à la houe ce qui s'appelle fouiller la terre. Le carré contient dix mille pas, de trois pieds $1 / 2$ de côté ; cette surface est un peu plus grande que celle de l'hectare. Mr Fleuriot le régisseur des Salines met un plant par pas, ce qui fait dix mille plants par carré, et pour assurer le succès de la plantation. » 
Bertrand trouve le temps de rédiger et de faire publier à Paris en 1838 un opuscule de 47 pages qu'il fait préfacer par le baron Charles Dupin: Sur la détresse des colonies françaises en général, de l'île Martinique en particulier consacré à l'étude de la production sucrière coloniale et la nécessité de diminuer la taxe sur le sucre exotique. (fig. $\left.\mathbf{n}^{\circ} \mathbf{1}\right)$ 


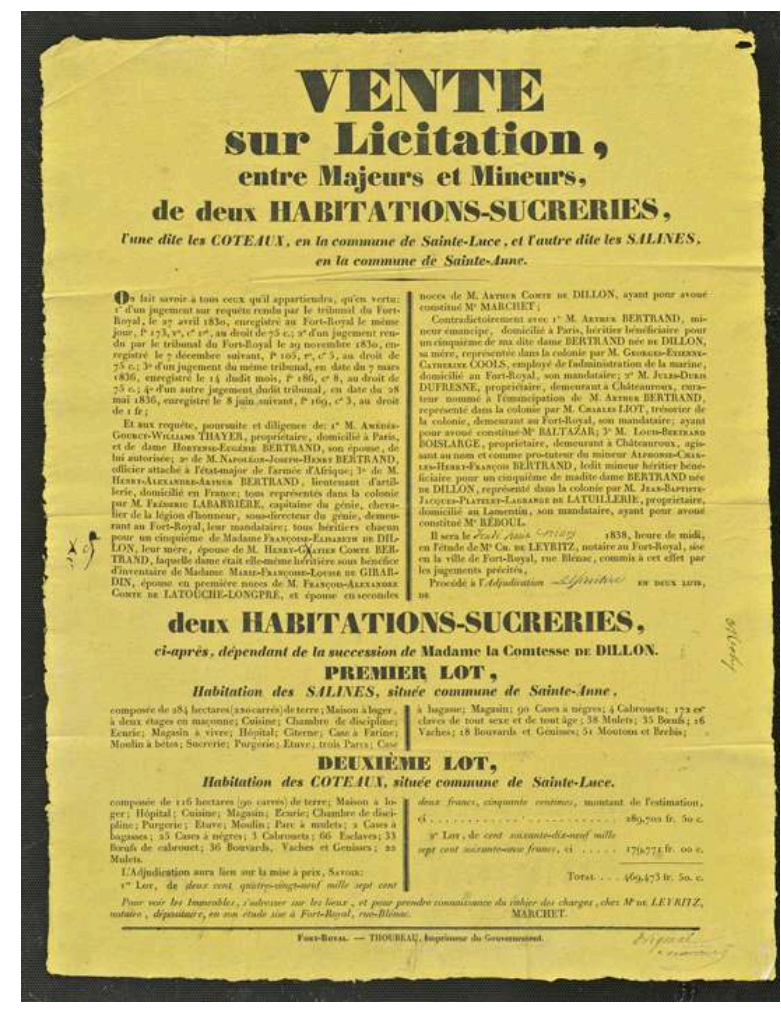

Vente de l'habitation Bertrand, affiche imprimée, 1838.

(c) FRANOM. Aix-en-Provence (183 APOM).

\section{Une affaire de famille : les Reiset}

Philibert Reiset ${ }^{2}$, alsacien né en 1777, arrive en Guadeloupe en 1801 comme commis de la Marine. Il va faire carrière dans l'administration : inspecteur du Trésor puis receveur et trésorier de la colonie. Dès 1804, il acquiert l'habitation sucrière "L'Espérance et Galard» (commune de Basse-Terre) qui compte 150 esclaves et couvre 150 ha, puis une habitation sucrière formée de deux plantations: Bovis (commune du Lamentin) et Métivier (commune de Sainte-Rose). L'ensemble couvre près de deux cents hectares et emploie environ deux cents esclaves. Ces terres proviennent des biens de Marie Françoise Bertille de Bovis, épouse de Jean-Baptiste César Dubuc de Saint-Olympe. Philibert Reiset gère mal ses affaires et, couvert de dettes, doit faire appel à son cousin Jacques Reiset, receveur général des finances du département de la Seine : en 1820, les deux hommes deviennent propriétaires, à titre d'antichrèse, de la propriété du Lamentin.

Jacques Reiset est un notable fortuné : alsacien lui aussi, né en 1771, il est nommé régent de la Banque de France en 1826; il est membre fondateur du Syndicat des receveurs généraux. Il est père de six enfants dont Jules (1818-1896) chimiste et agronome, député, et Jacques, receveur particulier du département de la Seine qui lui succédera dans les affaires.

21 En 1822, Jacques Reiset, qui ne s'entend guère avec son cousin, confie la gestion des plantations à Georges Giraud. La personnalité du gérant est remarquable : né en 1789 à Paris, il s'enrôle en 1807 et participe, comme cuirassier, aux campagnes 
napoléoniennes, dont la campagne de Russie avec le grade de sous-lieutenant. Il est blessé à plusieurs reprises et fait prisonnier en Russie de 1813 à 1814. Il participe à l'expédition du Sénégal de 1818, puis il passe commis de Marine en 1818, en poste à la Guadeloupe jusqu'en 1823. Il administre les terres des Reiset jusque dans les années 1840 puis, malade, rentre définitivement en France en 1846. Giraud joue un rôle déterminant dans l'histoire du domaine Reiset tant par ses qualités d'administrateur que par les relations privilégiées qu'il entretient avec l'ensemble de la famille.

Dans une de ses premières lettres du 15 août 1822, Jacques Reiset confie à Giraud: "Vous avez bien voulu m'offrir vos bons services et vous avez donné déjà tant de véritables marques d'attachement à la famille que je n'hésite pas à en réclamer un nouveau témoignage [...] je ne saurais en vérité m'adresser à personne dans la colonie avec le même abandon et la même confiance [...] ». Il lui demande d'abord de sortir Philibert de "son état de détresse »; ce dernier, par ses dettes, met la plantation en danger. «Je désire me tromper mais sa position me paraît désespérée » écrit Giraud en 1822 .

23 En 1823, Giraud prend les choses en mains : il lui faut rapidement relever la situation du Lamentin et effacer les traces de l'administration de Philibert Reiset qu'il qualifie de monstrueuse et désastreuse. Il adoucit le sort des esclaves, fait rentrer les marrons, encourage les naissances. «J'ai la satisfaction de voir beaucoup de nègres que je croyais perdus reprendre petit à petit leurs forces et leur santé par suite des soins et de ménagements que j'ai pour eux. » Jacques Reiset meurt en 1835, Philibert, rentré en France, meurt en 1836 ; les propriétés sont rapidement vendues aux enchères et acquises par les membres de la famille: Jacques Reiset et Eugène, fils de Philibert, qui sera délégué de la Guadeloupe à la Commission Schoelcher en 1846. Mais l'entente ne règne pas au sein de la famille; la gestion des terres confiée à plusieurs hommes d'affaires reste source de nombreux conflits.

Les archives de la famille Reiset constituent un ensemble exceptionnel d'archives de plantation tant par la qualité des documents produits que par leur quantité.

(fig. $\left.{ }^{\circ} 2\right)\left(\right.$ fig. $\left.n^{\circ} 3\right)\left(\right.$ fig. $\left.n^{\circ} 4\right)$ 
Figure 2

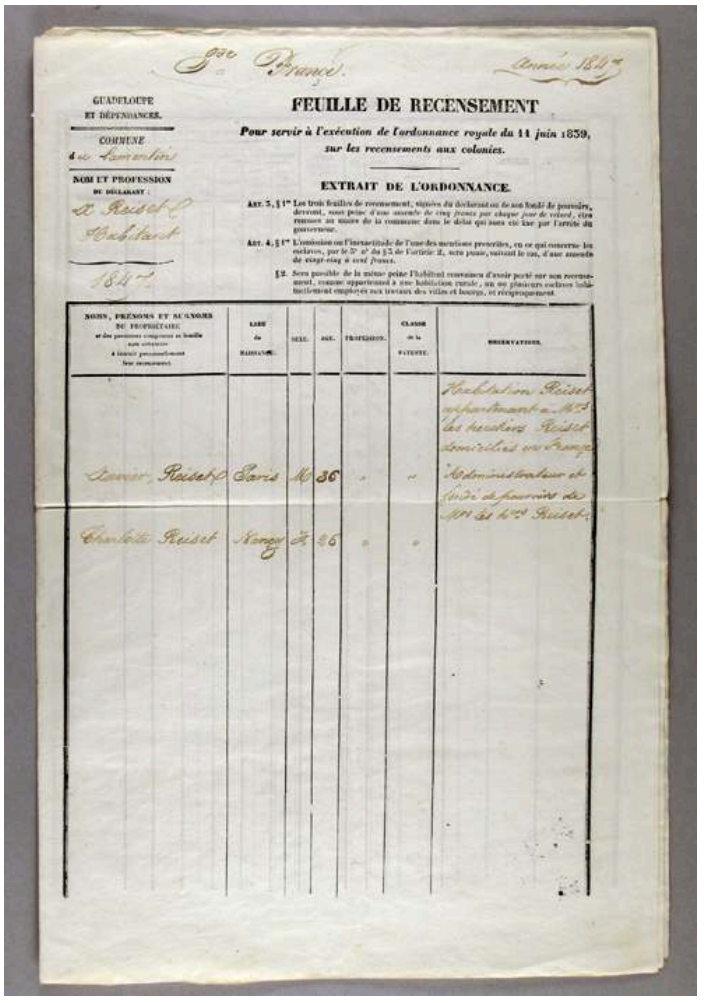

Plantation Reiset : dénombrement des esclaves, 1847. (c) FRANOM, Aix-en-Provence (170 APOM). 
Figure 3

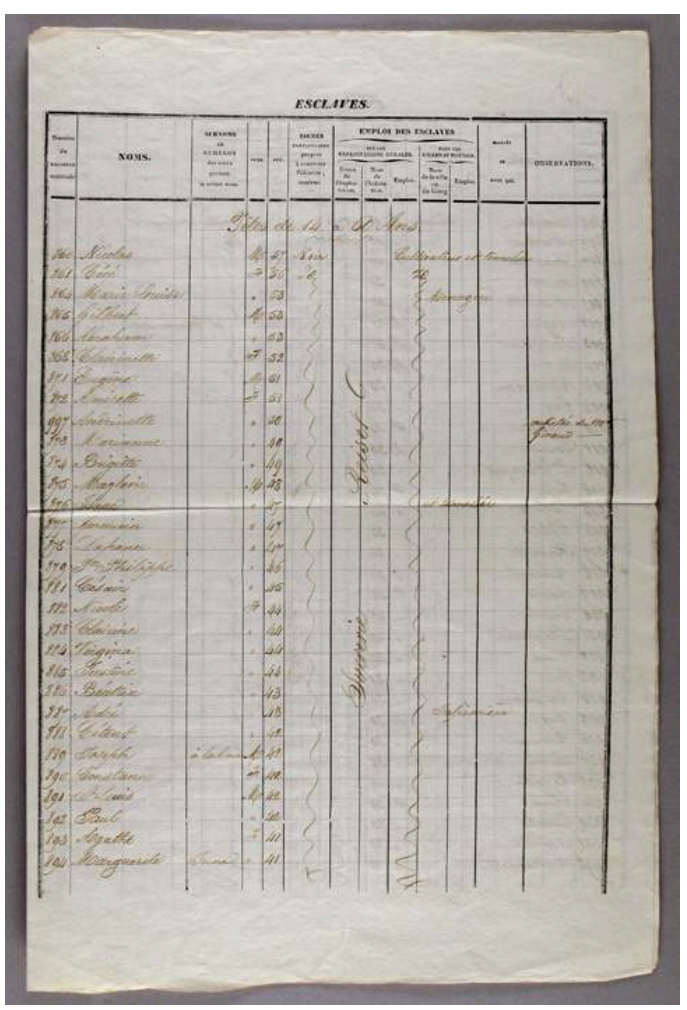

Plantation Reiset : dénombrement des esclaves, 1847. (c) FRANOM, Aix-en-Provence (170 APOM). 


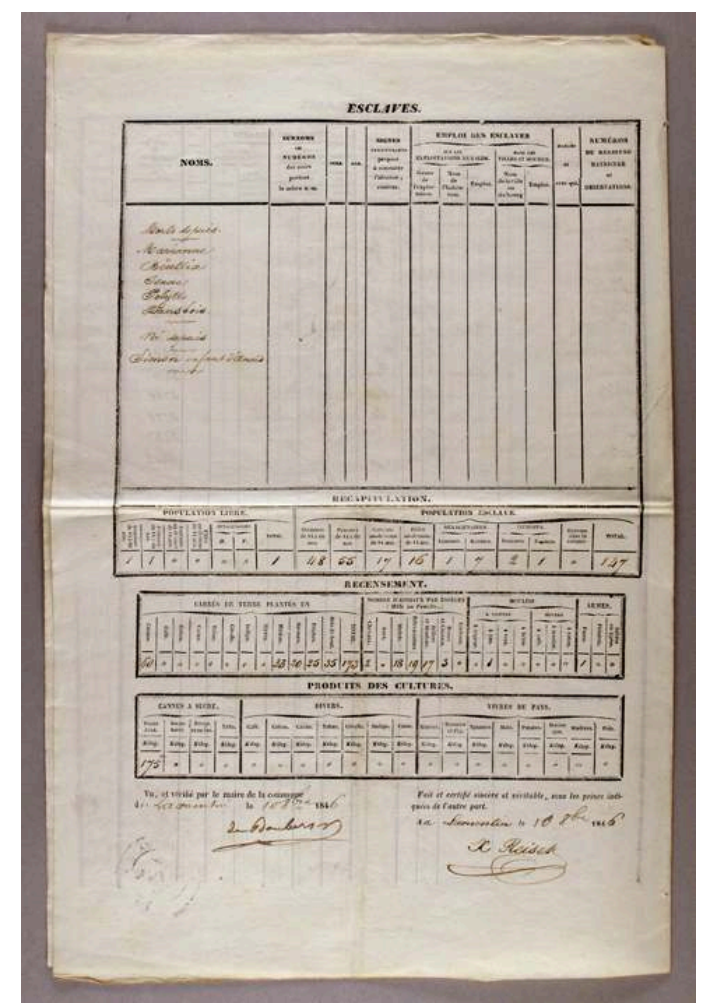

Plantation Reiset : dénombrement des esclaves, 1847.

(c) FRANOM, Aix-en-Provence (170 APOM).

27 Tous les documents les plus importants ont été préservés à commencer par la correspondance échangée entre les différents acteurs : des centaines de lettres depuis 1822 qui nous renseignent de façon détaillée sur la gestion des propriétés. L'aspect psychologique, largement présent, apporte un supplément d'information particulièrement précieux. Ces lettres sont d'autant plus passionnantes qu'elles émanent d'acteurs directs habiles à manier la plume, la correspondance du gérant Giraud est à cet égard une source d'informations sans égales.

On trouve aussi, parfaitement conservés : titres de propriétés, comptes des recettes et dépenses, journal quotidien de culture, recensements annuels des esclaves (depuis l'an XI jusqu'en 1847).

La période chronologique couvre les années 1820 à 1860 : les préoccupations des propriétaires sont dominées par la montée des idées abolitionnistes, les rapports difficiles avec l'administration publique. La fin de la traite a rendu plus forte la nécessité de garder la main d'œuvre en bonne santé et de veiller à son renouvellement « naturel ». L'obsession du gain et la crainte de l'endettement sont omniprésentes. Les vieilles peurs sont toujours là : empoisonnements, incendies, révoltes meurtrières, marronnage et désertions.

On ne saurait trop insister sur l'apport des archives de plantations, archives familiales et aussi archives économiques. Elles donnent accès à la société esclavagiste. Elles révèlent, au quotidien, les rouages complexes du monde des propriétaires terriens, traversé de tensions permanentes impliquant les relations de voisinage et les relations familiales. 
31 L'histoire des propriétés est directement concernée : les terres se morcellent; elles changent de mains et de noms. Leur situation juridique devient complexe et la plantation se révèle comme un lieu d'affrontements d'intérêts entre des héritiers, des ayants-droit, des créanciers.

Le gérant s'affirme comme personnage central impliqué dans un écheveau de rapports familiaux souvent conflictuels. Parfois délaissé, il doit dominer ces conflits, préserver les terres, les biens et les esclaves. Il lui faut faire durer le patrimoine, rendre des comptes et assurer les profits. Son témoignage est une source inestimable.

La mise à la disposition des chercheurs des papiers Bertrand et Reiset est un aboutissement particulièrement significatif dans la collecte et la préservation des sources de l'histoire de l'esclavage, de l'abolition et de ses conséquences.

\section{NOTES}

1. - Les papiers Bertrand ont été acquis par la Direction des Archives de France en 2008. Ils sont conservés aux Archives nationales d'outre-mer (Aix-en-Provence) sous la cote 183 APOM. Un certain nombre de textes ont été publiés dans l'ouvrage : CAMARA, Velyna, DION, Isabelle, DION, Jacques. Esclaves. Regards de blancs, 1672-1913. Collection Histoires d'outre-mer. Marseille : Images en manœuvres, Aix-en-Provence : Archives nationales d'outre-mer, 2008.

2. - Les papiers Reiset ont été acquis par la Direction des Archives de France en 2005 et 2006. Ils sont conservés aux Archives nationales d'outre-mer sous la cote 170 APOM. Un certain nombre de textes ont été publiés dans l'ouvrage : CAMARA, Velyna, DION, Isabelle, DION, Jacques. Esclaves. Regards de blancs, 1672-1913. Collection Histoires d'outre-mer. Marseille: Images en manœuvres, Aix-en-Provence : Archives nationales d'outre-mer, 2008.

\section{RÉSUMÉS}

L'auteur, à partir de deux exemples, explique l'intérêt historique des archives d'habitations sucrières esclavagistes et la nécessité de veiller à leur conservation.

Using two examples, the author explains the historical interest of sugar plantations' archives and the necessity of their conservation.

\section{INDEX}

Mots-clés : esclavage, plantations, sucre 
AUTEUR

JACQUES DION

Archives nationales d'outre-mer, jacques.dion@culture.gouv.fr 